\title{
Structure of graphene oxide: thermodynamics versus kinetics
}

\author{
Ning Lu, Zhenyu Li * and Jinlong Yang \\ Hefei National Laboratory for Physical Sciences at Microscale, \\ University of Science and Technology of China, Hefei, Anhui 230026, China
}

Graphene oxide (GO) is an important intermediate to prepare graphene and it is also a versatile material with various applications. However, despite its importance, the detailed structure of GO is still unclear. For example, previous theoretical studies based on energetics have suggested that hydroxyl chain is an important structural motif of GO, which, however, is found to be contrary to nuclear magnetic resonance (NMR) experiment. In this study, we check both thermodynamic and kinetic aspects missed previously. First principles thermodynamics gives a free energy based stability ordering similar to that based on energetics, and hydroxyl chain is thus thermodynamically still favorable. At the same time, by checking the calculated vibrational frequencies, we note that hydroxyl chain structure is also inconsistent with infrared experiment. Therefore, kinetics during GO synthesis is expected to make an important role in GO structure. Transition state calculations predict large energy barriers between local minima, which suggests that experimentally obtained GO has a kinetically constrained structure.

Recently, an intense research interest has been attracted by graphene oxide (GO). [1] Reduction of GO is a promising way to massively product graphene sample. 2-[5] At the same time, many applications of GO have been demonstrated, such as electronic device, [6 [8] chemical catalyst, [9, 10] hydrogen storage, [11, 12] and functional materials. [13, 14] Both to improve the sample quality of graphene from GO reduction and to better utilize GO as a new material, it is very desirable to understand the atomic details of GO structure.

A strong research effort has been devoted to the GO structure study, both theoretically [15 25] and experimentally. [5, 26 30] Based on nuclear magnetic resonance (NMR) experiment, it is believed that GO mainly has hydroxyl (-OH) and epoxy (-O-) groups on the basal plane and carboxylic acid groups $(-\mathrm{COOH})$ at edge sites. 26 28] Hydroxyl and epoxy groups are in close proximity, while $\mathrm{sp}^{2}$ carbon prefers to form small aromatic areas. [22, 27] More details about GO structure, such as possible structural motifs, are still under study.

Theoretically, many GO structure models based on small supercells have been constructed and asserted by comparing energies. [15, 16, 18, 20, 21] Important GO structure characteristics, such as the proximity of hydroxyl and epoxy groups, has been obtained in these studies. However, some controversies between theory and experiment exist. For example, although partially oxidized stable GO samples are routinely obtained in experiment, computational energetics suggests that fully covered GO is the most stable structure. 18 Another important conclusion from theoretical studies is that hydroxyl chain in GO is a very stable structure. [18 21] This result looks reasonable, since hydrogen bonds formed in this structure will strongly stabilize the system. However, our previous NMR simulation found that hydroxyl chain gives a too large ${ }^{13} \mathrm{C}$ chemical shift, and it thus should not be an important structural motif of GO. [22]

A possible reason for these discrepancies is from the thermodynamics: energetically favorable structure may not be the thermodynamically most stable one. For example, although GO is synthesized in solution, previous theoretical studies did not include solvation effects. Since hydroxyl group can also form hydrogen bond with solvent molecules, the stabilization effect of hydroxyl chain may be weakened by taking solution into account. Also, the phonon contribution to free energy has not been considered in previous theoretical studies, which may also alter the stability ordering. In this study, free energies of different GO structure models have been compared. However, the trend obtained from energetics is found to be qualitatively correct in the thermodynamics point of view. Therefore, we also consider the kinetics of GO structure evolution. We find that it is difficult to relax the GO structure from one local minimum to another. Therefore, GO may exist with a kinetically constrained structure, which explains the controversies between theory and experiment well.

Free energy $(G)$ is calculated with density functional theory (DFT) implemented in the DMOL ${ }^{3}$ package 31 , 32] under the generalized gradient approximation 33 ]

$$
G=E_{0}+\Delta G_{s o l}+E_{Z P E}+k_{B} T \sum_{i} \ln \left(1-e^{-\hbar \omega_{i} / k_{B} T}\right)
$$

where $\Delta G_{\text {sol }}$ is the free energy of solvation, and its electrostatic part is calculated using COMSO water solution model. [34, 35] $E_{Z P E}$ is the zero point energy correction, and $\omega_{i}$ is the phonon frequency at the zone center, which

${ }^{*}$ Corresponding author. E-mail: zyli@ustc.edu.cn 
is calculated by finite difference using a large supercell. Minimum-energy pathway for elementary reaction step is computed using the nudged elastic band (NEB) method [36] implemented in the Vienna Ab initio Simulation Package (VASP). 37, 38]

As a benchmark, we first consider isolated epoxy group and hydroxyl group using a $5 \times 5$ graphene supercell. With a single epoxy group, the optimized structure gives a $\mathrm{C}-\mathrm{O}$ bond length of $1.46 \AA$, and the distance between the two oxidized $\mathrm{C}$ atoms is $1.51 \AA$. The $\mathrm{O}-\mathrm{H}$ bond length in an isolated hydroxyl group is $0.98 \AA$, and the corresponding $\mathrm{C}-\mathrm{O}$ bond length is $1.49 \AA$. Vibrational frequency of $\mathrm{O}-\mathrm{H}$ stretch is $3659.1 \mathrm{~cm}^{-1}$ in the isolated hydroxyl group. Geometric parameters and vibrational frequency reported here agree well with previous study. [21] The calculated $\Delta G_{\text {sol }}$ is only $-0.08 \mathrm{eV}$ for isolated epoxy group, while it is $-0.25 \mathrm{eV}$ for isolated hydroxyl group, which is consistent with the stronger polarization of hydroxyl group.

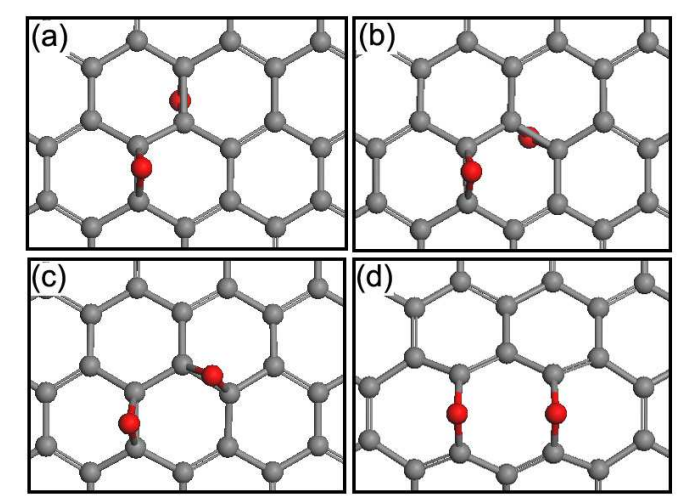

FIG. 1: Different configurations of two epoxy groups. A $5 \times 5$ supercell is using in calculation, which is not shown in the figure. Carbon is in gray and oxygen is in red.

To check the trends of energies and free energies ordering for different GO structures, we first consider two epoxy groups (2O). Four representative configurations including the two with lowest energies [20, 21] are studied (Figure 1). Their relative energies are listed in Table $1(1 \mathrm{a}-1 \mathrm{~d})$. Structures a and b have almost the same energy, which is 0.10 and $0.26 \mathrm{eV}$ lower than that of $\mathrm{c}$ and $\mathrm{d}$, respectively. This can be understood from a consideration of tension caused by epoxy groups. For structures a and b, tensions from both sides will compensate each other to decrease the total energy. Aligned epoxy groups prefer to break underlying C-C bonds, [17] as shown in Figure 1]. The solvation free energy varies from -0.08 to $-0.18 \mathrm{eV}$. With the large unit cell used here, zero point energy correction is more than 8 $\mathrm{eV}$, but their differences are small. Finally, the relative free energies at $300 \mathrm{~K}$ is similar to relative energies, with the same ordering.

We then study $2 \mathrm{OH}$ models (Figure 2) with two hydroxyl groups. Their relative energies are listed in Table 1) (2a2d). Our calculation shows the 1,2-hydroxyl pair has the lowest energy, which has been widely recognized in previous studies. 18, 19] Free energies follow the same order as energies, and their relative changes are small. In structures b and $\mathrm{d}$, hydrogen bonds are formed. The $\mathrm{O}-\mathrm{H}$ bonds involving in hydrogen bonds are slightly elongated, leading to two softer $\mathrm{O}-\mathrm{H}$ stretching mode with frequencies about $3450 \mathrm{~cm}^{-1}$. For other $\mathrm{O}-\mathrm{H}$ bonds, the corresponding frequencies are between 3640 and $3720 \mathrm{~cm}^{-1}$.

Considering the proximity of hydroxyl and epoxy groups in real GO structure, we discuss several typical $\mathrm{O}+2 \mathrm{OH}$ models (Figure 3). Their relative energies are listed in Table \ (3a-3d). In structure a, two hydroxyl groups are attached to carbon atoms directly adjacent to epoxide at the opposite side of the carbon plane, which is the most stable. Structure b, with an epoxy group and a neighboring 1,2-hydroxyl pair, has almost the same energy (0.03 eV higher). Structures c and d have much higher energies. Calculated free energies have the same trend. Hydrogen bond induced softening of $\mathrm{O}-\mathrm{H}$ stretching mode is also observed similar to the $2 \mathrm{OH}$ case.

Besides these isolated-oxidation-group models, we also consider fully oxidized models, which goes to another limit. The fully oxidized graphene epoxide (O-full) has two configurations, which are shown in Figure 4 a and b. With a $2 \times 3$ supercell for structure a and a $2 \times 2$ supercell for structure b, we have the same number of atoms in both structures. The calculated relative energy and relative free energy are still very similar.

Two models with 100\% hydroxyl coverage (OH-full) are constructed. One (Figure 4 ) contains hydroxyl chains, while the other (Figure 4d) does not. As shown in Table I (4c-4d), structure c with hydroxyl chains is $0.13 \mathrm{eV}$ lower in energy. Its free energy is also $0.07 \mathrm{eV}$ lower. Strong hydrogen bonds in structure c elongate O-H bonds to about 
TABLE I: Energy $\left(E_{0}\right)$, solvation free energy $\left(\Delta G_{s o l}\right)$, zero point correction $\left(E_{Z P E}\right)$, and free energy at $300 \mathrm{~K}(G(300))$ of different GO models. $E_{0}$ and $G(300)$ are listed with the most stable configuration as the reference. All values are in eV.

\begin{tabular}{cccccc}
\hline \hline \multicolumn{1}{c}{ 2O } & & $E_{0}$ & $\Delta G_{\text {sol }}$ & $E_{Z P E}$ & $G(300)$ \\
\hline & 1a & 0.00 & -0.14 & 8.72 & 0.00 \\
& 1b & 0.00 & -0.16 & 8.75 & 0.02 \\
& 1c & 0.10 & -0.18 & 8.69 & 0.04 \\
& 1d & 0.26 & -0.08 & 8.70 & 0.35 \\
\hline $2 \mathrm{OH}$ & 2a & 0.00 & -0.30 & 9.37 & 0.00 \\
& 2b & 0.40 & -0.24 & 9.30 & 0.39 \\
& 2c & 0.70 & -0.31 & 9.30 & 0.61 \\
& 2d & 1.37 & -0.39 & 9.27 & 1.19 \\
\hline O+2OH & 3a & 0.00 & -0.28 & 9.45 & 0.00 \\
& 3b & 0.03 & -0.26 & 9.44 & 0.03 \\
& 3c & 0.68 & -0.37 & 9.45 & 0.57 \\
& 3d & 0.86 & -0.46 & 9.39 & 0.61 \\
\hline O-full & 4a & 0.00 & -0.34 & 5.12 & 0.00 \\
& 4b & 3.25 & -0.39 & 5.08 & 3.17 \\
\hline OH-full & 4c & 0.00 & -0.03 & 2.33 & 0.00 \\
& 4d & 0.13 & -0.02 & 2.30 & 0.07 \\
\hline O-OH-full & 5a & 0.00 & -0.19 & 4.02 & 0.00 \\
& 5b & 0.19 & -0.19 & 4.03 & 0.20 \\
\hline O-OH-sp & 5c & 0.00 & -0.18 & 3.83 & 0.00 \\
& 5d & 0.12 & -0.24 & 3.84 & 0.03 \\
\hline \hline
\end{tabular}
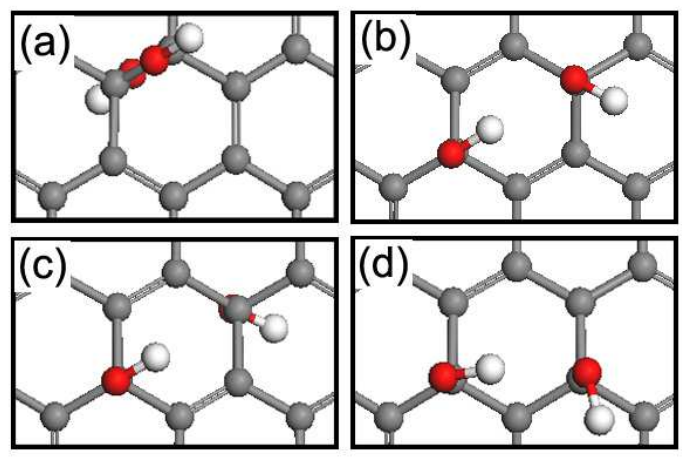

FIG. 2: Different configurations of the two hydroxyl groups. A $5 \times 5$ supercell is used in calculation, which is not shown in the figure. Carbon is in gray, oxygen is in red, and hydrogen is in white.

$1.0 \AA$ and decrease $\mathrm{O}-\mathrm{H}$ stretching frequencies to $3050-3270 \mathrm{~cm}^{-1}$. In contrast, $\mathrm{O}-\mathrm{H}$ stretching in structure $\mathrm{d}$ has frequencies from 3360 to $3500 \mathrm{~cm}^{-1}$.

Structure a in Figure 5 is a stable fully-oxidized GO model (O-OH-full) with hydroxyl chains proposed by Yan et al. 18] based on energetics, which is $0.19 \mathrm{eV}$ lower in energy than structure b without hydroxyl chain. When free energy is considered instead, structure a is still $0.20 \mathrm{eV}$ more stable than structure b. O-H bonds in hydroxyl chain are elongated to about $1.01 \AA$ due to the formed hydrogen bonds. Corresponding $\mathrm{O}-\mathrm{H}$ stretching frequencies are generally between $2800-3100 \mathrm{~cm}^{-1}$. These frequencies for structure $\mathrm{b}$ are around $3450 \mathrm{~cm}^{-1}$. Since the experiment value of $\mathrm{O}-\mathrm{H}$ stretching frequency of GO is about $3400 \mathrm{~cm}^{-1}$ [39, 40] or higher [41], our frequency calculations also suggested that hydroxyl chain is not a GO structure motif.

As another test, partially oxidized structures $\left(\mathrm{O}-\mathrm{OH}-\mathrm{sp}^{2}\right)$ with the ratio of $\mathrm{C}\left(\mathrm{sp}^{2}\right) / \mathrm{C}(-\mathrm{O}-) / \mathrm{C}(-\mathrm{OH})$ equal to $1: 1: 1$ are considered (Figure 5 and 5 $\mathrm{d})$. As shown in Table $1(5 \mathrm{c}-5 \mathrm{~d})$ ), structure $\mathrm{c}$ with hydroxyl chains is $0.12 \mathrm{eV}$ lower in energy than structure d and $0.04 \mathrm{eV}$ lower in free energy. Although the difference becomes smaller when free energy 


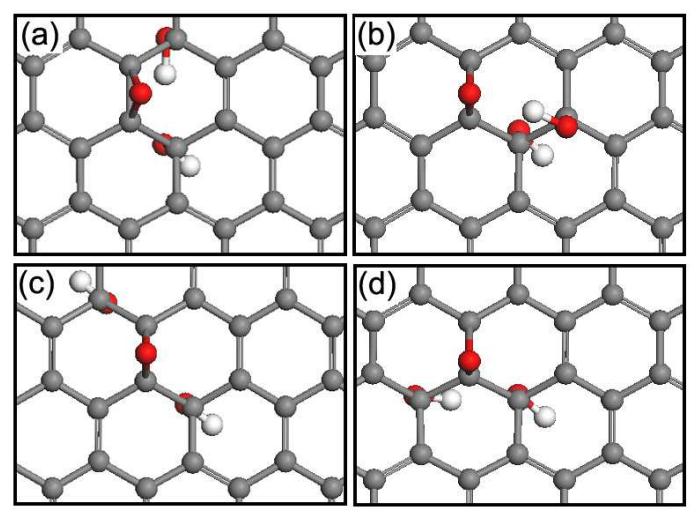

FIG. 3: Different configurations of one epoxy group plus two hydroxyl groups. A $5 \times 5$ supercell is used in calculation, which is not shown in the figure. Carbon is in gray, oxygen is in red, and hydrogen is in white.

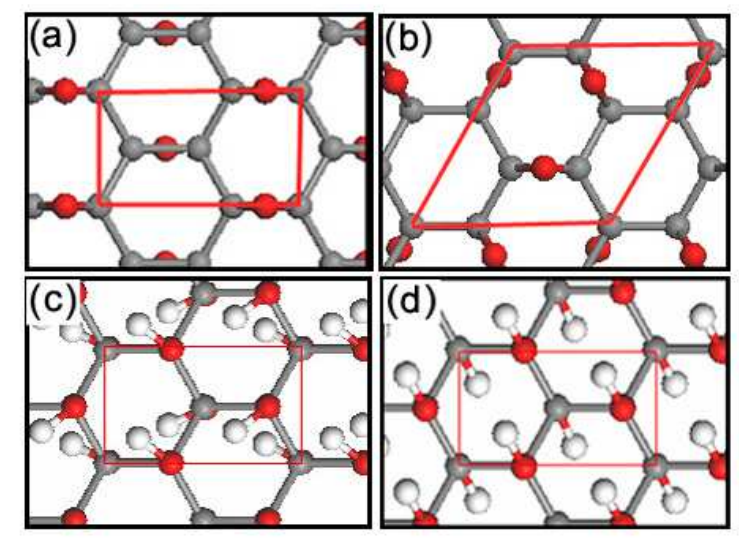

FIG. 4: (a) and (b) Different configurations of fully oxidized graphene epoxide. (c) and (d) Different configurations of fully oxidized GO with hydroxyl groups only. The unit cell used in calculation is marked in red. Carbon is in gray, oxygen is in red, and hydrogen is in white.

is considered, it is safe to conclude that hydroxyl chain is thermodynamically still a very stable structure. The O-H stretching frequencies in structure c are among 2800-3100 $\mathrm{cm}^{-1}$, much smaller than experimental value. Those in structure d are around $3580 \mathrm{~cm}^{-1}$.

Since hydroxyl chain gives too large chemical shift and too small O-H stretching frequency compared to experimental values, while it is still very stable with both solvation and phonon contributions to free energy taken into account, it is more likely the formation of hydroxyl chain is prohibited by kinetics during the formation of GO. To check the possibility of experimentally obtained GO as a metastable state, we consider the oxidation-group diffusion in GO.

As shown in Figure 6a, isolated hydroxyl group is easy to diffuse on pristine graphene surface [24] with an energy barrier only $0.27 \mathrm{eV}$. The $\mathrm{C}-\mathrm{O}$ distance is $1.51 \AA$ in the initial state and it is elongated to $2.51 \AA$ in the transition state. When we add an additional neighboring hydroxyl group as shown in Fig 6 b, the diffusion barrier increases to $1.13 \mathrm{eV}$. Therefore, the diffusion barrier of hydroxyl group strongly depends on its environment. It is thus important to check the hydroxyl mobility in a realistic GO model with a certain epoxy and hydroxyl group concentration. With the model shown in Figure [6;, the diffusion barrier becomes as large as $2.89 \mathrm{eV}$. This is because that a stable GO structure requires a delicate balance between positions of hydroxyl and epoxy groups to minimize stress. Typically, moving a hydroxyl group at the same time fixing other groups will lead to a large change in energy. In our case, the finial state is $2.57 \mathrm{eV}$ higher in energy than the initial state, which leads to a very high diffusion barrier.

The diffusion barrier for an isolated epoxy group is $0.74 \mathrm{eV}$, similar to the value $(0.9 \mathrm{eV})$ obtained by Li et al. previously with cluster models. [42] When a neighboring epoxy group is added (Figure 7b), the diffusion barrier becomes $0.40 \mathrm{eV}$, which also demonstrates a strong environment dependence. Similar to the hydroxyl diffusion case, 


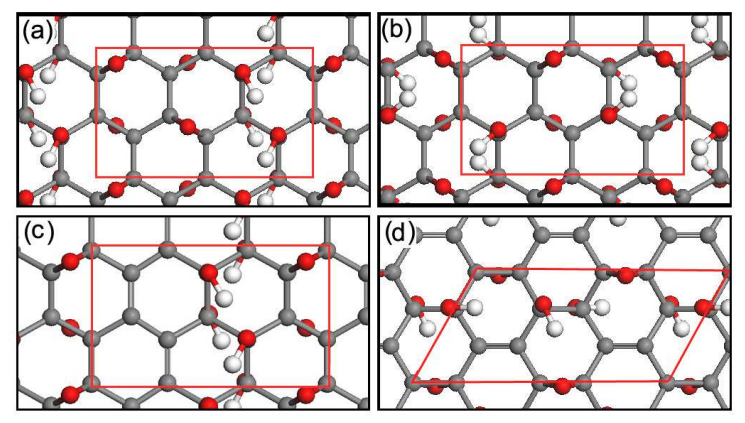

FIG. 5: (a)and (b) Different configurations of fully oxidized GO with both epoxy and hydroxyl groups. (c) and (d) Different configurations of partially oxidized GO with both epoxy and hydroxyl groups. The unit cell used in calculation is marked in red. Carbon is in gray, oxygen is in red, and hydrogen is in white.

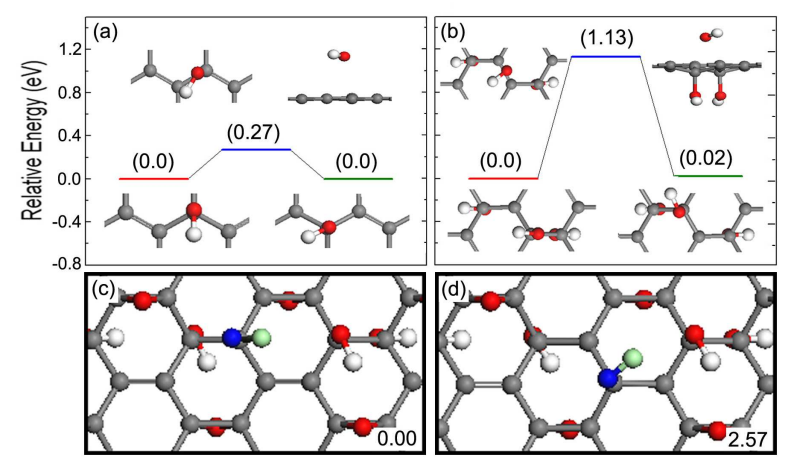

FIG. 6: (a) and (b) Energy profile for hydroxyl diffusion. Insets are optimized structure of the initial, transition, and final states. A $5 \times 5$ supercell is used in calculation, which is not shown in the figure. (c) Initial state of hydroxyl diffusion in a partially oxidized GO, and its corresponding (d) final state, where relative energies are marked. Carbon is in gray, oxygen is in red, and hydrogen is in white. In (c) and (d), atoms involving in the diffusion in marked in different colors (blue for $\mathrm{O}$ and green for $\mathrm{H}$ ).

in a realistic GO model (Figure 7 ) $)$, a very large diffusion barrier $(2.0 \mathrm{eV})$ is obtained due to the energy difference between the initial and final states.

Exchange of a hydroxyl group and a neighboring epoxy group can be realized by a $\mathrm{H}$ diffusion between them. There are two different kinds of transition states, which leads to different final states. The first kind of transition states occupy 1,2-sites (Figure 8 8 ) and the second one is on 1,3-sites (Figure 8 $\mathrm{b}$ ). In the first case, the diffusion barrier is very small $(0.18 \mathrm{eV})$, as also reported previously with cluster models. [12] In the second case, we obtain a much higher diffusion barrier of $0.88 \mathrm{eV}$. The longer distance between $\mathrm{H}$ and $\mathrm{O}$ in the transition state leads to higher diffusion barrier. When a realistic GO model is used, the diffusion barrier increase to 1.33 and $1.95 \mathrm{eV}$ for the 1,2 and 1,3-cases, respectively.

According to our calculations, although it is possible to design some delicate structures, in which the barrier of oxidation group diffusion is low, generally the diffusion barrier is very high at least in one direction. Therefore, a global relaxation of GO structure is very difficult. GO may easily relax to a local minimum, as evidenced also by molecular dynamics simulations, [23] but different local minima are expected to be separated by large barriers. Experimentally available GO thus typically has a kinetically constrained metastable structure, which explains why spectroscopic signal of thermodynamically stable hydroxyl chain structure is not observed in experiment. This result can also explain why homogenous phase is formed in partially oxidized GO, although phase separated structure is more stable. 20]

In summary, both thermodynamic and kinetic aspects of GO structure have been considered from first principles. By taking the solvation effect and phonon contribution into account, we calculate the free energies of different GO structure models. We find that hydroxyl chain is really a very stable structure. However, the calculated vibrational frequencies and also previously obtained chemical shifts of this structure is not observed in infrared and NMR experiments. Therefore, kinetics during the synthesis of GO must have made an important role in GO structure. We then study 


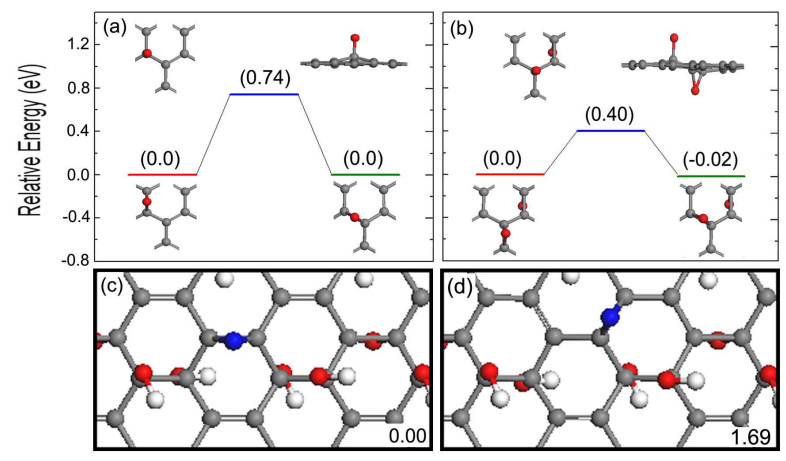

FIG. 7: (a) and (b) Energy profile for epoxy group diffusion. Insets are optimized structure of the initial, transition, and final states. A $5 \times 5$ supercell is used in calculation, which is not shown in the figure. (c) Initial state of epoxy group diffusion in a partially oxidized GO, and its corresponding (d) final state, where relative energies are marked. Carbon is in gray, oxygen is in red, and hydrogen is in white. In (c) and (d), atoms involving in the diffusion in marked in different colors (blue for O and green for $\mathrm{H})$.

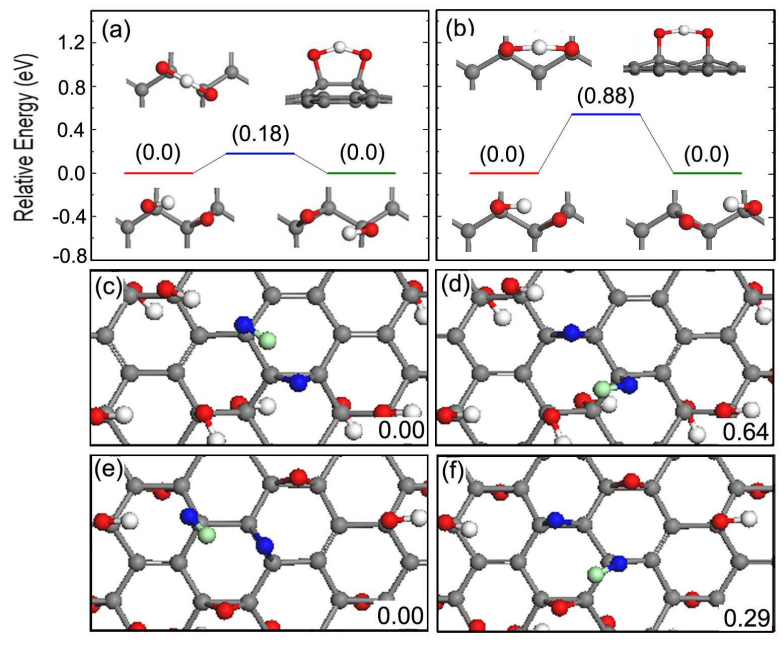

FIG. 8: (a) and (b) Energy profile for H diffusion from a hydroxyl group to a neighboring epoxy group. Insets are optimized structure of the initial, transition, and final states. A $5 \times 5$ supercell is used in calculation, which is not shown in the figure. (c) Initial state of $\mathrm{H}$ diffusion with 1,2-transition state in a partially oxidized GO, and its corresponding (d) final state, where relative energies are marked. (e) Initial state of $\mathrm{H}$ diffusion with 1,3-transition state in a partially oxidized GO, and its corresponding (f) final state, where relative energies are marked. Carbon is in gray, oxygen is in red, and hydrogen is in white. In (c) and (d), atoms involving in the diffusion in marked in different colors (blue for $\mathrm{O}$ and green for $\mathrm{H}$ ).

the diffusion of oxidation groups in GO. Typically, there is a very large diffusion barrier. Therefore, experimentally available GO will has a kinetically constrained structure. To understand GO structure better, more studies on the oxidation process of graphite is highly desirable.

Acknowledgements. The authors are grateful to Prof. Zhi-Pan Liu for helpful discussion. This work is partially supported by NSFC (20933006 and 20803071), by MOE (FANEDD-2007B23 and NCET-08-0521), by MOST (2011CB921404), by CAS (KJCX2-YW-W22), and by USTC-SCC, SCCAS, and Shanghai Supercomputer Centers.

[1] D. R. Dreyer, S. Park, C. W. Bielawski and R. S. Ruoff, Chem. Soc. Rev., 2010, 39, 228.

[2] S. Stankovich, D. A. Dikin, G. H. B. Dommett, K. M. Kohlhaas, E. J. Zimney, E. A. Stach, R. D. Piner, S. T. Nguyen and R. S. Ruoff, Nature (London), 2006, 442, 282.

[3] S. Gilje, S. Han, M. Wang, K. L. Wang and R. B. Kaner, Nano Lett., 2007, 7, 3394.

[4] D. Li, M. B. Muller, S. Gilje, R. B. Kaner and G. G. Wallace, Nat. Nanotechnol., 2008, 3, 101. 
[5] W. Gao, L. B. Alemany, L. Ci and P. M. Ajayan, Nature Chemistry, 2009, 1, 403.

[6] X. Wu, M. Sprinkle, X. Li, F. Ming, C. Berger and W. A. de Heer, Phys. Rev. Lett., 2008, $101,026801$.

[7] S. Wang, J. Pu, D. S. H. Chan, B. J. Cho and K. P. Loh, Appl. Phys. Lett., 2010, 96, 143109.

[8] W. K. Kim, Y. M. Jung, J. H. Cho, J. Y. Kang, J. Y. Oh, H. Kang, H.-J. Lee, J. H. Kim, S. Lee, H. J. Shin, J. Y. Choi, S. Y. Lee, Y. C. Kim, I. T. Han, J. M. Kim, J.-G. Yook, S. Baik and S. C. Jun, Appl. Phys. Lett., $2010,97,193103$.

[9] G. M. Scheuermann, L. Rumi, P. Steurer, W. Bannwarth and R. Mülhaupt, J. Am. Chem. Soc., $2009,131,8262$.

[10] G. Eda, Y.-Y. Lin, C. Mattevi, H. Yamaguchi, H.-A. Chen, I.-S. Chen, C.-W. Chen and M. Chhowalla, Adv. Mater., 2010, 22, 505.

[11] L. Wang, F. H. Yang and R. T. Yang, Ind. Eng. Chem. Res. 2009, 48, 2920.

[12] G. M. Psofogiannakis and G. E. Froudakis, J. Am. Chem. Soc., 2009, 131, 15133.

[13] D. A. Dikin, S. Stankovich, E. J. Zimney, R. D. Piner, G. H. B. Dommett, G. Evmenenko, S. T. Nguyen and R. S. Ruoff, Nature(London), 2007, 448, 457.

[14] J. W. Burress, S. Gadipelli, J. Ford, J. M. Simmons, W. Zhou and T. Yildirim, Agnew. Chem. Int. Ed. 2010, 49, 8902-8904.

[15] D. W. Boukhvalov and M. I. Katsnelson, J. Am. Chem. Soc., 2008, 130, 10697.

[16] R. J. W. E. Lahaye, H. K. Jeong, C. Y. Park and Y. H. Lee, Phys. Rev. B, 2009, 79, 125435.

[17] Z. Li, W. Zhang, Y. Luo, J. Yang and J. G. Hou, J. Am. Chem. Soc., 2009, 131, 6320.

[18] J. A. Yan, L. Xian and M. Y. Chou, Phys. Rev. Lett., 2009, 103, 086802.

[19] L. Wang, K. Lee, Y.-Y. Sun, M. Lucking, Z. Chen, J. J. Zhao and S. B. Zhang, ACS Nano, $2009,3,2995$.

[20] L. Wang, Y. Y. Sun, K. Lee, D. West, Z. F. Chen, J. J. Zhao and S. B. Zhang, Phys. Rev. B, $2010,82,161406$.

[21] J. A. Yan and M. Y. Chou, Phys. Rev. B, 2010, 82, 125403.

[22] N. Lu, Y. Huang, H. Li, Z. Li and J. Yang, J. Chem. Phys., 2010, 133, 034502.

[23] J. T. Paci, T. Belytschko, and G. C. Schatz, J. Phys. Chem. C, 2007, 111, 18099.

[24] N. Ghaderi and M. Peressi, J. Phys. Chem. C, 2011, 114, 21625.

[25] W. Zhang, V. Carravetta, Z. Li, Y. Luo and J. Yang, J. Chem. Phys., 2009, 131, 244505.

[26] A. Lerf, H. He, M. Forster and J. Klinowski, J. Phys. Chem. B, 1998, 102, 4477.

[27] W. Cai, R. D. Piner, F. J. Stadermann, S. Park, M. A. Shaibat, Y. Ishii, D. Yang, A. Velamakanni, S. J. An, M. Stoller, J. An, D. Chen and R. S. Ruoff, Science, 2008, 321, 1815.

[28] L. B. Casabianca, M. A. Shaibat, W. W. Cai, S. Park, R. Piner, R. S. Ruoff, and Y. Ishii, J. Am. Chem. Soc., 2010, 132, 5672.

[29] S. Saxena, T. A. Tyson and E. Negusse J. Phys. Chem. Lett. 2010, 1, 3433.

[30] D. W. Lee, L. De Los Santos V., J. W. Seo, L. L. Felix, A. Bustamante D., J. M. Cole and C. H. W. Barnes, J. Phys. Chem. B 2010, 114, 5723.

[31] B. Delley, J. Chem. Phys., 1990, 92, 508.

[32] B. Delley, J. Chem. Phys., 2003, 113, 7756.

[33] J. P. Perdew, Burke, K. and M. Ernzerhof, Phys. Rev. Lett., 1996, 77, 3865.

[34] A. Klamt and G. Schüürmann, J. Chem. Soc., Perkin Trans. 2, 1993, 5, 799.

[35] B. Delley, Mol. Simul., 2006, 32, 117.

[36] G. Henkelman and H. Jonsson, J. Chem. Phys., 2000, 113, 9978.

[37] G. Kresse and J. Furthmuller, Comput. Mater. Sci., 1996, 6, 15.

[38] G. Kresse and J. Furthmuller, Phys. Rev. B, 1996, 54, 11169.

[39] E. Choi, T, H. Han, J. Hong, E. J. Kim, S. H. Lee, H. W. Kim and S. O. Kim, J. Mater. Chem., $2010,20,1907$.

[40] C. Nethravathi, J. T. Rajamathi, N. Ravishankar, C. Shivakumara and M. Rajamathi, Langmuir, $2008,24,8240$.

[41] T. Szabó, O. Berkesi, P. Forgó, K. Josepovits, Y. Sanakis, D. Petridis and I. Dékány, Chem. Mater. 2006, 18, $2740-2749$.

[42] J. Li, K. N. Kudin, M. J. McAllister, R. K. Prudhomme, I, A. Aksay and R. Car, Phys. Rev. Lett., $2006,96,176101$. 